\title{
Efficacy of Cultural Method of Weed Control on Growth, Yield and Quality of Linseed (Linum usitatissimum L.)
}

\author{
H.J. Chhaganiya, A.P. Patel* and V.D. Patel \\ Department of Agronomy, N.M. College of Agriculture, Navsari Agricultural University, \\ Navsari 396 450, Gujarat, India \\ *Corresponding author
}

\section{A B S T R A C T}

\section{Keywords \\ Linseed, Oxyfluorfen, Isoproturon, Quizalofop ethyl, Weed management \\ Article Info \\ Accepted: \\ 24 January 2018 \\ Available Online: \\ 10 February 2018}

A field experiment was conducted during rabi season of 2014-2015 at the College Farm, N. M. College of Agriculture, Navsari Agricultural University, Navsari to study the effect of weed management practices on growth, yield and quality of linseed (Linum usitatissimum L.). Growth parameters, yield attributes, oil yield and seed yield were higher with lower weed count and weed dry weight in treatment $\left(\mathrm{T}_{11)}\right.$ weed free (interculturing followed by hand weeding at 20 and 40 DAS).

\section{Introduction}

Linseed or flax is among the oldest crop plants cultivated for the purpose of oil and fiber. It belongs to the genus Linum and family Linaceae. The botanical name, Linum usitatissimum was given by Linnaeus in his book "Species Plantarum" (Linnaeus, C., 1857). It is an annual herbaceous plant with shallow root system. The common names flax and linseed are used in North America and Asia, respectively, for $L$. usitatissimum. Oilseed varieties and fiber varieties are specialized development of this species (Millam et al., 2005). The cultivars grown primarily for seed/oil purpose are relatively short in height and possess more secondary branches and seed bolls (seed capsule). The cultivars grown for fiber purpose are tall growing with straight culms and have fewer secondary branches.

The present weed control practices are characterized by intensive use of manual labour and animal power. Both of them are in short supply and increasingly became uneconomical. Adverse soil and climatic conditions prevent timely removal of weeds through manual and mechanical means. Linseed having less branching habit, small leaf area and show growth during initial growth period, it complete poorly with weeds and often suffers from severe weed competition. Unchecked weed growth has 
been reported to reduce grain yield of linseed to the tune of 34.2 per cent (Mani et al., 1968).

Every part of linseed plant is utilized commercially, either directly or after processing. Seed contains 33 to 47 per cent oil. A small quantity is directly used for edible purposes. About 20 per cent of the total oil produced is used at farmer level and the rest 80 per cent oil goes to industries in various forms, such as boiled oil, borated oil, eposidized oil, aluminated oil, urethane oil, isomerized oil etc. Seeds of linseed contain high levels of dietary fibers as well as lignans, an abundance of micronutrients and omega-3 fatty acids. The oil $(>66 \%)$ is rich in linolenic acid and is a perfect drying oil. The seed of linseed content nutrient value per $100 \mathrm{~g}$ is carbohydrates $28.88 \mathrm{~g}$, sugars $1.55 \mathrm{~g}$, fat 42.16 $\mathrm{g}$, protein $18.29 \mathrm{~g}$ and dietary fibers $27.39 \mathrm{~g}$ (Anonymous, 2013).

\section{Materials and Methods}

Experiment was conducted on the farm of college of Agriculture, Navsari during rabi season of 2014-2015. The linseed crop local variety was sown on alkaline soil having $(\mathrm{pH}$ 7.8). The experiment was conducted in Randomized Block Design with twelve treatments replicated three times. The treatment comprises of four herbicides viz., Pendimethalin and Oxyfluorfen as preemergence and Isoproturon and Quizalofop ethyl as a post-emergence with or without cultural practices. There was conventional method of weed control like manual weeding and interculturing for comparison of effect.

The treatment details are $\left(\mathrm{T}_{1}\right)$ Pendimethalin @ $750 \mathrm{~g} / \mathrm{ha}$ as pre-emergence, $\left(\mathrm{T}_{2}\right)$ Oxyfluorfen @ 60 g/ha as pre-emergence, $\left(\mathrm{T}_{3}\right)$ Isoproturon@ @ $750 \mathrm{~g} / \mathrm{ha}$ as post-emergence, ( $\mathrm{T}_{4}$ ) Quizalofop ethyl @ $75 \mathrm{~g} / \mathrm{ha}$ as postemergence, $\left(\mathrm{T}_{5}\right) \mathrm{T}_{1} \mathrm{fb}$ IC and HW at 30 DAS, $\left(\mathrm{T}_{6}\right) \mathrm{T}_{2} \mathrm{fb}$ IC and HW at $30 \mathrm{DAS},\left(\mathrm{T}_{7}\right) \mathrm{T}_{3} \mathrm{fb}$ IC and $\mathrm{HW}$ at $30 \mathrm{DAS},\left(\mathrm{T}_{8}\right) \mathrm{T}_{4} \mathrm{fb}$ IC and $\mathrm{HW}$ at 30 DAS, $\left(\mathrm{T}_{9}\right)$ Pendimethalin $\left(\mathrm{T}_{1}\right)+$ Quizalofop ethyl $\left(\mathrm{T}_{4}\right),\left(\mathrm{T}_{10}\right) 1 \mathrm{HW}$ at $20 \mathrm{DAS}$, $\left(\mathrm{T}_{11}\right)$ Weed free (IC fb by HW at 20 and 40 DAS) and $\left(\mathrm{T}_{12}\right)$ Weedy check. Linseed local variety was sown on 20 November 2014 at 30 $\mathrm{x} 10 \mathrm{~cm}$ spacing. Observation regarding growth study i.e. plant height and number of branches per plant were carried out at 30,60, 90 DAS and at harvest. Weed study i.e. weed count (No. per $\mathrm{m}^{2}$ ) at 25, $50 \mathrm{DAS}$ and at harvest. Dry weight of weeds $(\mathrm{kg} / \mathrm{ha})$ were determined at harvest. While yield attributing characters i.e. number of capsules plant $^{-1}$, number of seeds capsules plant $^{-1}$ and seed yield and stover yield were noted at harvest.

\section{Results and Discussion}

\section{Weeds}

Weed flora identified in experiment plot were monocot, Dicot and Sedges weeds. In monocot weeds Cynodon dactylon, Digitaria sanguinalist, Bracharia spp, Sorghum halepense, Echinochloa crusgallis and in dicot weeds Alternanthera sessillis, Physalis minima, Euphorbia hirta, Vernonia cinerea, Amaranthus viridis, Digera arvensis were observed while under sedges weed Cyperus rotundus were observed.

Effect of weed management practices on weed count and dry weight of weed of weeds

At 25, 50 DAS and at harvest stage weed count (monocot + dicot + sedges) and dry weight of weeds at harvest were recorded and are presented in Table 1. All weed control treatments recorded significant reduction in weed count of weeds and dry weight of weeds. The treatments $\mathrm{T}_{11}$ Weed free (IC followed by hand weeding at 20 and 40 DAS) recorded significantly the lowest number of monocot, dicot and sedges weeds at 25, 50 DAS and at 
harvest but remained at par with $\mathrm{T}_{10}(1 \mathrm{HW}$ at 20 DAS), $\mathrm{T}_{5}$ ( $\mathrm{T}_{1}$ fb IC and HW at 30 DAS) and $\mathrm{T}_{1}$ pendimethalin @ $750 \mathrm{~g} \mathrm{ha}^{-1}$ as preemergence at $25 \mathrm{DAS}$. The treatment $\mathrm{T}_{10}$ (1 HW at 20 DAS) recorded significantly the lowest number of monocot, dicot and sedges weeds next to $\mathrm{T}_{11}(1.46,1.33$ and 1.46 per cent $\mathrm{m}^{2}$ ). It clearly indicated that IC followed by 1 HW significantly reduced the weed population during initial period of crop growth.

At 50 DAS and at harvest, besides treatment $\mathrm{T}_{11}$ weed free (interculturing followed by hand weeding at 20 and 40 DAS), $\mathrm{T}_{5}, \mathrm{~T}_{8}$ and $\mathrm{T}_{6}$ recorded significantly lower number of monocot, dicot and sedge weeds as compare to the treatment weedy check $\left(\mathrm{T}_{12}\right)$. The removal of weed at regular interval through hand weeding at 20 and 40 DAS accounted for less count of monocot, dicot and sedge weeds under treatment $T_{11}$. Similarly, effective control of monocot, dicot and sedge weeds by integration of herbicides with hand weeding was responsible for lower number of monocot, dicot and sedge weeds under treatments $\mathrm{T}_{5}, \mathrm{~T}_{8}$ and $\mathrm{T}_{6}$. At harvest treatment $\mathrm{T}_{5}, \mathrm{~T}_{8}$ and $\mathrm{T}_{6}$ was at par with treatment $\mathrm{T}_{11}$ weed free (interculturing followed by hand weeding at 20 and 40 DAS) for monocot, dicot and sedge weeds.

The treatment weedy check $\left(\mathrm{T}_{12}\right)$ recorded the high population of monocot, dicot and sedge weeds owing to uncontrolled condition. Tomar et al., (1990) reported significant reduction in grassy and broad leaved weeds which causes 37.9 per cent reduction in yield of linseed. The lower dry weight of weeds were recorded and presented in (Table 2) at harvest (248.85 $\mathrm{kg} / \mathrm{ha}$ ) with treatment $\mathrm{T}_{11}$ weed free (interculturing followed by hand weeding at 20 and 40 DAS) due to hand weeding. Owing to fact that hand weeding cause a substantial reduction in weed density hence, recorded the lowest dry weight of weeds. It was statistically at par with treatments $T_{5}, T_{8}$ and $T_{6}$ at harvest.

\section{Weed control efficiency and weed index}

Reduction in dry weight of weed accumulation leads to maximization of weed control efficiency. With the application of treatment $\mathrm{T}_{11}$ Weed free (interculturing followed by hand weeding at 20 and 40 DAS) recorded highest weed control efficiency 84.09 per cent was clearly recorded in (Table 2) which was followed by treatment $T_{5}$ and $T_{8}$ having weed control efficiency of 81.60 and 80.90 per cent at harvest respectively. While, minimum weed index $(0.00 \%)$ was recorded under treatment $\mathrm{T}_{11}$ Weed free (interculturing followed by hand weeding at 20 and 40 DAS). Frisen and freer (1991) reported that only pre emergence herbicides application was less effective and allow weed competition in later stages, therefore along with cultural practices (hoeing or weeding) or post emergence herbicides gave maximum weed control efficiency.

\section{Effect of weed management practices on growth and development of linseed}

Plant height $(\mathrm{cm})$ and Number of branches per plant were recorded at 30, 60, 90 DAS and at harvest presented in Table 3 . Treatment $\left(\mathrm{T}_{11}\right)$ Weed free (IC followed by HW at 20 and 40 DAS) was observed highest plant height $(19.46 \mathrm{~cm})$ which was statistically at par with $\mathrm{T}_{5}, \mathrm{~T}_{6}$ and $\mathrm{T}_{8}$ at 30 DAS. The highest plant height $(42.30,55.49$ and $63.06 \mathrm{~cm})$ was observed under treatment $\left(\mathrm{T}_{11}\right)$ Weed free (IC followed by $\mathrm{HW}$ at 20 and $40 \mathrm{DAS}$ ) and was statistically at par with $\mathrm{T}_{5}, \mathrm{~T}_{8}$, and $\mathrm{T}_{6}$ at 60,90 and at harvest. While more number of branches per plant (8.01 and 8.43) was recorded with treatment $\left(\mathrm{T}_{11}\right)$ Weed free (IC followed by $\mathrm{HW}$ at 20 and $40 \mathrm{DAS}$ ) at 90 (DAS) and at harvest, but it was found statistically at par with treatments $\mathrm{T}_{5}, \mathrm{~T}_{6}$, and $\mathrm{T}_{8}$ (90 DAS) and $\mathrm{T}_{5}, \mathrm{~T}_{6}$, and $\mathrm{T}_{8}$ at harvest. It is established fact that weed compete for light, space, nutrient and water with the crop and hamper overall growth of the same. 
Table.1 Effect of weed management practices on monocot, dicot and sedges weed density at 25, 50 DAS and at harvest

\begin{tabular}{|c|c|c|c|c|c|c|c|c|c|}
\hline \multirow[b]{2}{*}{ Treatments } & \multicolumn{3}{|c|}{ Monocot weed $\left(\mathrm{m}^{-2}\right)$} & \multicolumn{3}{|c|}{ Dicot weed $\left(\mathrm{m}^{-2}\right)$} & \multicolumn{3}{|c|}{ Sedge weed $\left(\mathrm{m}^{-2}\right)$} \\
\hline & 25 DAS & 50 DAS & $\begin{array}{c}\text { At } \\
\text { Harvest }\end{array}$ & 25 DAS & 50 DAS & $\begin{array}{c}\text { At } \\
\text { Harvest }\end{array}$ & 25 DAS & 50 DAS & $\begin{array}{c}\text { At } \\
\text { harvest }\end{array}$ \\
\hline $\begin{array}{l}\mathbf{T}_{1:} \text { Pendimethalin @ } 750 \mathrm{~g} / \mathrm{ha} \text { as } \\
\text { PE }\end{array}$ & $\begin{array}{c}1.68 \\
(2.33)\end{array}$ & $\begin{array}{l}3.67 \\
(13)\end{array}$ & $\begin{array}{c}5.46 \\
(29.33)\end{array}$ & $\begin{array}{c}1.68 \\
(2.33)\end{array}$ & $\begin{array}{l}4.84 \\
(23)\end{array}$ & $\begin{array}{c}6.16 \\
(37.66)\end{array}$ & $\begin{array}{c}1.53 \\
(1.88)\end{array}$ & $\begin{array}{c}6.16 \\
(37.66)\end{array}$ & $\begin{array}{l}7.15 \\
(51)\end{array}$ \\
\hline $\mathbf{T}_{2:}$ Oxyfluorfen @ $60 \mathrm{~g} / \mathrm{ha}$ as PE & $\begin{array}{c}2.11 \\
(4)\end{array}$ & $\begin{array}{c}3.85 \\
(14.33)\end{array}$ & $\begin{array}{c}5.72 \\
(32.33)\end{array}$ & $\begin{array}{c}2.20 \\
(4.33)\end{array}$ & $\begin{array}{l}5.23 \\
(27)\end{array}$ & $\begin{array}{c}6.40 \\
(40.66)\end{array}$ & $\begin{array}{c}2.34 \\
(5)\end{array}$ & $\begin{array}{l}6.27 \\
(39)\end{array}$ & $\begin{array}{c}7.48 \\
(56.66)\end{array}$ \\
\hline T3:Isoproturon@ @750 g/ha as PoE & $\begin{array}{c}3.89 \\
(14.66)\end{array}$ & $\begin{array}{l}4.40 \\
(19)\end{array}$ & $\begin{array}{c}6.25 \\
(38.66)\end{array}$ & $\begin{array}{c}4.12 \\
(16.66)\end{array}$ & $\begin{array}{l}5.51 \\
(30)\end{array}$ & $\begin{array}{c}6.96 \\
(48.33)\end{array}$ & $\begin{array}{c}4.44 \\
(19.33)\end{array}$ & $\begin{array}{c}6.48 \\
(41.66)\end{array}$ & $\begin{array}{l}7.83 \\
(61)\end{array}$ \\
\hline $\begin{array}{l}\text { T4:Quizalofopethyl @ } 75 \mathrm{~g} / \mathrm{ha} \text { as } \\
\text { PoE }\end{array}$ & $\begin{array}{c}3.84 \\
(14.33)\end{array}$ & $\begin{array}{c}3.62 \\
(12.66)\end{array}$ & $\begin{array}{l}5.60 \\
(31)\end{array}$ & $\begin{array}{c}4.00 \\
(15.66)\end{array}$ & $\begin{array}{c}4.25 \\
(17.66)\end{array}$ & $\begin{array}{c}6.21 \\
(38.33)\end{array}$ & $\begin{array}{l}3.92 \\
(15)\end{array}$ & $\begin{array}{l}5.86 \\
(34)\end{array}$ & $\begin{array}{c}7.31 \\
(53.33)\end{array}$ \\
\hline $\mathbf{T}_{5:} \mathrm{T}_{1} \mathrm{fb} \mathrm{IC}$ and $\mathrm{HW}$ at $30 \mathrm{DAS}$ & $\begin{array}{c}1.56 \\
(2.66)\end{array}$ & $\begin{array}{c}2.03 \\
(3.66)\end{array}$ & $\begin{array}{c}3.43 \\
(11.33)\end{array}$ & $\begin{array}{c}1.52 \\
(2)\end{array}$ & $\begin{array}{c}1.95 \\
(3.33)\end{array}$ & $\begin{array}{c}3.26 \\
(10.33)\end{array}$ & $\begin{array}{c}1.52 \\
(1.81)\end{array}$ & $\begin{array}{c}3.07 \\
(9)\end{array}$ & $\begin{array}{c}5.17 \\
(26.33)\end{array}$ \\
\hline $\mathbf{T}_{6:} \mathrm{T}_{2} \mathrm{fb} \mathrm{IC}$ and $\mathrm{HW}$ at $30 \mathrm{DAS}$ & $\begin{array}{c}1.95 \\
(3.33)\end{array}$ & $\begin{array}{c}2.19 \\
(4.33)\end{array}$ & $\begin{array}{c}3.62 \\
(12.66)\end{array}$ & $\begin{array}{c}2.48 \\
(5.66)\end{array}$ & $\begin{array}{c}2.10 \\
(4)\end{array}$ & $\begin{array}{l}3.37 \\
(11)\end{array}$ & $\begin{array}{c}2.96 \\
(8.33)\end{array}$ & $\begin{array}{l}3.23 \\
(10)\end{array}$ & $\begin{array}{l}5.32 \\
(28)\end{array}$ \\
\hline $\mathbf{T}_{7:} \mathrm{T}_{3} \mathrm{fb} \mathrm{IC}$ and $\mathrm{HW}$ at $30 \mathrm{DAS}$ & $\begin{array}{c}4.02 \\
(15.66)\end{array}$ & $\begin{array}{c}2.79 \\
(7.33)\end{array}$ & $\begin{array}{l}4.33 \\
(17)\end{array}$ & $\begin{array}{l}4.18 \\
(17)\end{array}$ & $\begin{array}{c}2.60 \\
(6.33)\end{array}$ & $\begin{array}{l}4.54 \\
(20)\end{array}$ & $\begin{array}{c}3.58 \\
(12.33)\end{array}$ & $\begin{array}{c}4.12 \\
(16.66)\end{array}$ & $\begin{array}{c}6.16 \\
(37.66)\end{array}$ \\
\hline $\mathbf{T}_{8}: \mathrm{T}_{4} \mathrm{fb} \mathrm{IC}$ and $\mathrm{HW}$ at $30 \mathrm{DAS}$ & $\begin{array}{c}3.76 \\
(13.33)\end{array}$ & $\begin{array}{c}2.11 \\
(4)\end{array}$ & $\begin{array}{c}3.57 \\
(12.33)\end{array}$ & $\begin{array}{l}4.04 \\
(16)\end{array}$ & $\begin{array}{c}2.03 \\
(3.33)\end{array}$ & $\begin{array}{c}3.32 \\
(10.66)\end{array}$ & $\begin{array}{c}3.48 \\
(11.66)\end{array}$ & $\begin{array}{c}3.17 \\
(9.66)\end{array}$ & $\begin{array}{l}5.22 \\
(27)\end{array}$ \\
\hline $\begin{array}{l}\text { T:Pendimethalin }\left(\mathrm{T}_{1}\right)+ \\
\text { Quizalofopethyl }\left(\mathrm{T}_{4}\right)\end{array}$ & $\begin{array}{c}1.54 \\
(2.33)\end{array}$ & $\begin{array}{c}2.91 \\
(8)\end{array}$ & $\begin{array}{c}5.78 \\
(33.33)\end{array}$ & $\begin{array}{c}1.77 \\
(2.66)\end{array}$ & $\begin{array}{c}2.73 \\
(6)\end{array}$ & $\begin{array}{c}4.65 \\
(21.33)\end{array}$ & $\begin{array}{c}1.53 \\
(1.90)\end{array}$ & $\begin{array}{c}5.66 \\
(31.66)\end{array}$ & $\begin{array}{c}7.27 \\
(52 . .66)\end{array}$ \\
\hline $\mathbf{T}_{10:} 1 \mathrm{HW}$ at $20 \mathrm{DAS}$ & $\begin{array}{c}1.46 \\
(1.66)\end{array}$ & $\begin{array}{c}5.46 \\
(29.33)\end{array}$ & $\begin{array}{c}7.32 \\
(53.33)\end{array}$ & $\begin{array}{c}1.34 \\
(1.33)\end{array}$ & $\begin{array}{c}4.87 \\
(23.33)\end{array}$ & $\begin{array}{c}7.24 \\
(52.33)\end{array}$ & $\begin{array}{c}1.46 \\
(1.66)\end{array}$ & $\begin{array}{c}7.98 \\
(63.33)\end{array}$ & $\begin{array}{c}8.47 \\
(71.66)\end{array}$ \\
\hline $\begin{array}{l}\text { T.11: Weed free (IC follow by HW } \\
\text { at } 20 \text { and } 40 \text { DAS) }\end{array}$ & $\begin{array}{c}1.34 \\
(1.33)\end{array}$ & $\begin{array}{c}1.85 \\
(3)\end{array}$ & $\begin{array}{l}3.38 \\
(11)\end{array}$ & $\begin{array}{c}1.22 \\
(1)\end{array}$ & $\begin{array}{c}1.76 \\
(2.66)\end{array}$ & $\begin{array}{c}2.90 \\
(8)\end{array}$ & $\begin{array}{c}1.34 \\
(1.33)\end{array}$ & $\begin{array}{c}2.85 \\
(7.66)\end{array}$ & $\begin{array}{c}4.80 \\
(22.66)\end{array}$ \\
\hline $\mathbf{T}_{12:}$ Weedy check & $\begin{array}{c}5.27 \\
(27.33)\end{array}$ & $\begin{array}{c}6.94 \\
(47.66)\end{array}$ & $\begin{array}{l}8.96 \\
(80)\end{array}$ & $\begin{array}{c}5.11 \\
(25.66)\end{array}$ & $\begin{array}{l}7.63 \\
(58)\end{array}$ & $\begin{array}{c}8.54 \\
(72.66)\end{array}$ & $\begin{array}{c}6.31 \\
(39.33)\end{array}$ & $\begin{array}{l}9.23 \\
(80)\end{array}$ & $\begin{array}{c}10.41 \\
(108.33)\end{array}$ \\
\hline S. Em. \pm & 0.13 & 0.19 & 0.31 & 0.20 & 0.28 & 0.40 & 0.16 & 0.31 & 0.44 \\
\hline C.D. at $5 \%$ & 0.39 & 0.55 & 0.91 & 0.59 & 0.82 & 1.17 & 0.48 & 0.92 & 1.30 \\
\hline C. V. \% & 8.56 & 9.45 & 10.21 & 12.44 & 12.85 & 13.10 & 9.96 & 10.18 & 11.23 \\
\hline
\end{tabular}


Table.3 Effect of integrated weed management practices on plant height $(\mathrm{cm})$ and number of branches per plant of linseed

\begin{tabular}{|c|}
\hline Treatments \\
\hline $\mathrm{T}_{1}$ :Pendimethalin@ $950 \mathrm{~g} / \mathrm{ha}$ as PE \\
\hline $\mathrm{T}_{2:}$ Oxyfluorfen @ $60 \mathrm{~g} / \mathrm{ha}$ as PE \\
\hline 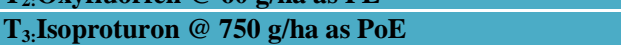 \\
\hline 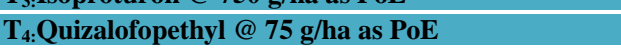 \\
\hline $\mathrm{T}_{5:} \mathrm{T}_{1} \mathrm{fb}$ IC and HW at $30 \mathrm{DAS}$ \\
\hline $\mathrm{T}_{6:} \mathrm{T}_{2} \mathrm{fb} \mathrm{IC}$ and $\mathrm{HW}$ at $30 \mathrm{DAS}$ \\
\hline $\mathrm{T}_{7}: \mathrm{T}_{3} \mathrm{fb} \mathrm{IC}$ and $\mathrm{HW}$ at $30 \mathrm{DAS}$ \\
\hline $\mathrm{T}_{8:} \mathrm{T}_{4} \mathrm{fb} \mathrm{IC}$ and $\mathrm{HW}$ at $30 \mathrm{DAS}$ \\
\hline$T_{9:}$ Pendimethalin $\left(T_{1}\right)+$ Quizalofopethyl $\left(T_{4}\right)$ \\
\hline $\mathrm{T}_{10:} 1 \mathrm{HW}$ at $20 \mathrm{DAS}$ \\
\hline$T_{11:}$ Weed free (IC follow by HW at 20 and 40 DAS) \\
\hline $\mathrm{T}_{12:}$ Weedy check \\
\hline S. Em. \pm \\
\hline C.D. at $5 \%$ \\
\hline C. V. \% \\
\hline
\end{tabular}

\begin{tabular}{|c|c|c|c|c|c|c|c|}
\hline \multicolumn{9}{|c|}{ Plant height $(\mathbf{c m})$} & \multicolumn{5}{|c|}{ Number of branches plant } \\
\hline 30 DAS & $\mathbf{6 0}$ DAS & $\mathbf{9 0}$ DAS & At harvest & 30 DAS & 60 DAS & 90 DAS & At harvest \\
\hline 17.37 & 37.36 & 48.07 & 55.59 & 2.23 & 4.57 & 5.67 & 6.66 \\
\hline 17.27 & 36.13 & 47.56 & 55.15 & 1.85 & 4.51 & 5.34 & 6.56 \\
\hline 16.80 & 36.54 & 45.76 & 52.54 & 1.71 & 4.45 & 4.67 & 6.33 \\
\hline 16.98 & 37.66 & 46.25 & 53.65 & 1.73 & 4.34 & 5.01 & 6.59 \\
\hline 18.30 & 39.66 & 53.68 & 61.59 & 2.11 & 5.31 & 7.67 & 7.89 \\
\hline 18.13 & 38.82 & 51.63 & 60.90 & 1.98 & 4.92 & 7.34 & 7.65 \\
\hline 17.14 & 37.45 & 49.53 & 55.98 & 2.14 & 4.73 & 6.34 & 6.86 \\
\hline 17.80 & 39.60 & 52.18 & 61.19 & 1.87 & 5.12 & 7.01 & 7.53 \\
\hline 17.33 & 37.26 & 48.32 & 55.78 & 1.85 & 4.54 & 6.01 & 6.84 \\
\hline 17.41 & 32.05 & 43.73 & 51.41 & 1.77 & 4.60 & 4.34 & 5.50 \\
\hline 19.46 & 42.30 & 55.49 & 63.06 & 2.25 & 5.40 & 8.01 & 8.43 \\
\hline 15.63 & 31.35 & 41.11 & 49.65 & 1.84 & 3.64 & 4.01 & 5.00 \\
\hline 0.69 & 1.50 & 1.99 & 2.36 & 0.12 & 0.33 & 0.45 & 0.52 \\
\hline 2.02 & 4.40 & 5.86 & 6.95 & $\mathbf{N S}$ & $\mathbf{N S}$ & 1.33 & 1.55 \\
\hline 6.85 & 7.00 & 7.12 & 7.28 & 11.49 & 12.53 & 13.25 & 13.45 \\
\hline
\end{tabular}

Table.4 Effect of different treatments on yield attributing characters, quality and yield of linseed

\begin{tabular}{|c|c|c|c|c|c|c|c|}
\hline \multirow[t]{2}{*}{ Treatments } & \multicolumn{3}{|c|}{ Yield attributing characters } & \multicolumn{2}{|c|}{ Yield } & \multicolumn{2}{|c|}{ Quality parameters } \\
\hline & $\begin{array}{c}\text { Number of } \\
\text { capsules per } \\
\text { plant }\end{array}$ & $\begin{array}{l}\text { Number of } \\
\text { seeds per } \\
\text { capsule }\end{array}$ & $\begin{array}{l}1000 \text { Seed } \\
\text { weight } \\
\text { (g) }\end{array}$ & $\begin{array}{l}\text { Seed yield } \\
\text { (kg/ha) }\end{array}$ & $\begin{array}{c}\text { Stover yield } \\
\text { (kg/ha) }\end{array}$ & $\begin{array}{l}\text { Oil content } \\
(\%)\end{array}$ & $\begin{array}{c}\text { Oil yield } \\
\text { (kg/ha) }\end{array}$ \\
\hline $\mathrm{T}_{1:}$ Pendimethalin@ $750 \mathrm{~g} / \mathrm{ha}$ as PE & 47.54 & 7.13 & 6.33 & 668 & 2056 & 37.00 & 248 \\
\hline $\mathrm{T}_{2:}$ Oxyfluorfen @ $60 \mathrm{~g} / \mathrm{ha}$ as PE & 46.08 & 7.10 & 6.25 & 642 & 2039 & 37.00 & 238 \\
\hline $\mathrm{T}_{3:}$ Isoproturon@ $950 \mathrm{~g} / \mathrm{ha}$ as PoE & 44.43 & 6.97 & 6.14 & 631 & 2024 & 36.93 & 234 \\
\hline $\mathrm{T}_{4:}$ Quizalofopethyl @ $75 \mathrm{~g} / \mathrm{ha}$ as PoE & 45.84 & 7.03 & 6.14 & 657 & 2053 & 36.90 & 243 \\
\hline $\mathrm{T}_{5:} \mathrm{T}_{1} \mathrm{fb} \mathrm{IC}$ and $\mathrm{HW}$ at 30DAS & 52.54 & 8.07 & 6.65 & 801 & 2358 & 38.03 & 305 \\
\hline $\mathrm{T}_{6:} \mathrm{T}_{2} \mathrm{fb} \mathrm{IC}$ and $\mathrm{HW}$ at $30 \mathrm{AS}$ & 50.44 & 7.83 & 6.53 & 720 & 2220 & 37.73 & 272 \\
\hline $\mathrm{T}_{7:} \mathrm{T}_{3} \mathrm{fb} \mathrm{IC}$ and $\mathrm{HW}$ at 30DAS & 49.75 & 7.37 & 6.37 & 711 & 2206 & 37.33 & 265 \\
\hline $\mathrm{T}_{8:} \mathrm{T}_{4} \mathrm{fb} \mathrm{IC}$ and $\mathrm{HW}$ at $30 \mathrm{DAS}$ & 50.32 & 7.60 & 6.37 & 798 & 2351 & 37.00 & 296 \\
\hline $\mathrm{T}_{9:}$ Pendimethalin $\left(\mathrm{T}_{1}\right)+$ Quizalofopethyl $\left(\mathrm{T}_{4}\right)$ & 48.68 & 7.15 & 6.41 & 688 & 2061 & 37.50 & 258 \\
\hline $\mathrm{T}_{10} 1 \mathrm{HW}$ at $20 \mathrm{DAS}$ & 41.28 & 6.63 & 6.02 & 635 & 2045 & 36.33 & 230 \\
\hline $\mathrm{T}_{11}$ : Weed free (IC follow by HW at 20 and 40 DAS) & 56.01 & 8.40 & 6.76 & 810 & 2391 & 38.40 & 310 \\
\hline$T_{12:}$ Weedy check & 36.71 & 5.33 & 6.26 & 510 & 1982 & 35.60 & 183 \\
\hline S. Em. \pm & 2.20 & 0.41 & 0.22 & 33.74 & 111.68 & 2.17 & 16.81 \\
\hline C.D. at $5 \%$ & 6.46 & 1.23 & NS & 98.97 & 327.57 & NS & 49.32 \\
\hline C. V. \% & 8.04 & 10.06 & 6.24 & 8.47 & 9.00 & 10.14 & 11.32 \\
\hline
\end{tabular}


Table.2 Dry weight of weed, weed control efficiency and weed index as influenced by different weed management treatments

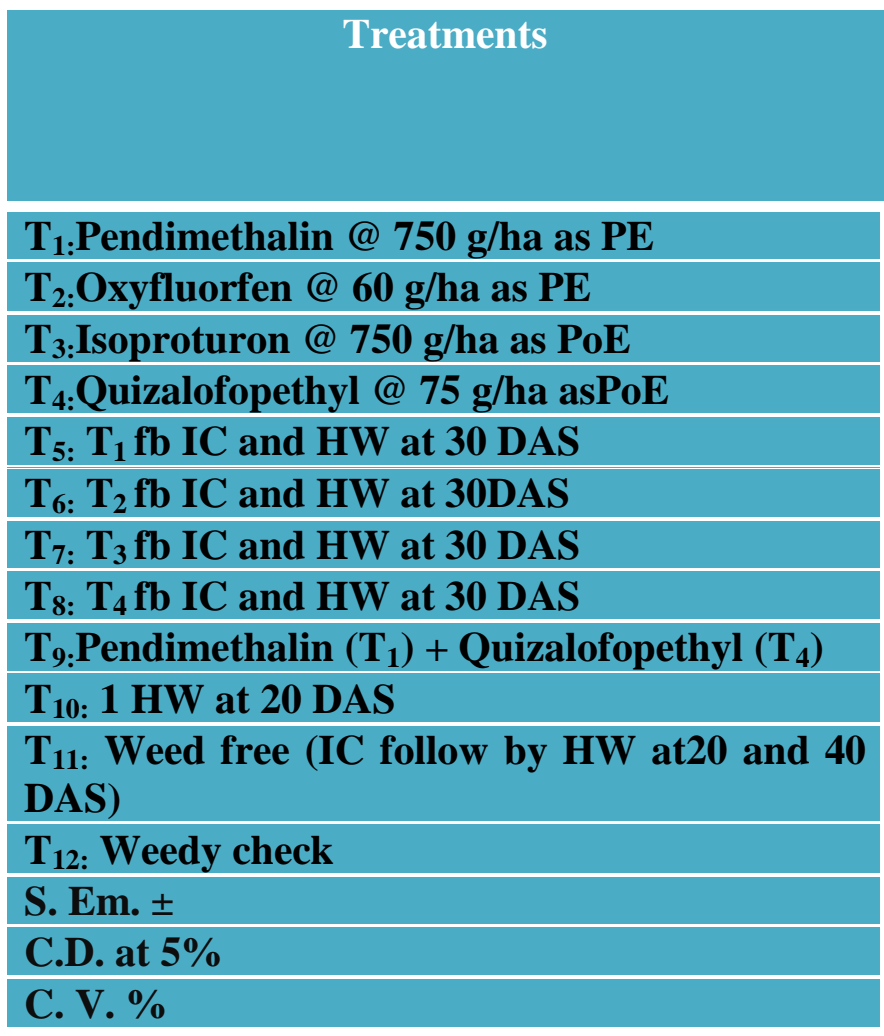

If weeds are removed by weed control methods, the trend was reversed and crop gain height as well as more number of branches per plant.

\section{Effect of weed management practices on yield attributing characters and yield}

Yield attributes as number of capsules plant ${ }^{-1}$ and number of seeds capsules $^{-1}$ noted significant increase under treatment $\mathrm{T}_{11}$ weed free (IC follow by $\mathrm{HW}$ at 20 and 40 DAS) being at par with $\mathrm{T}_{5}, \mathrm{~T}_{6}, \mathrm{~T}_{8}$ and $\mathrm{T}_{7}$. But in case of test weight did not significant due to different weed management treatments. While Seed and Stover yield (Table 4) were found significantly higher under treatment $\mathrm{T}_{11}$ weed free (interculturing followed by hand weeding at 20 and 40 DAS) but was remained at par with treatments $\mathrm{T}_{5}\left(\mathrm{~T}_{1} \mathrm{fb}\right.$ IC and $\mathrm{HW}$ at 30 DAS), $\mathrm{T}_{8}$ ( $\mathrm{T}_{4} \mathrm{fb}$ IC and HW at $\left.30 \mathrm{DAS}\right), \mathrm{T}_{6}$

\begin{tabular}{|c|c|c|}
\hline $\begin{array}{c}\text { Dry weight of } \\
\text { weeds at } \\
\text { harvest } \\
(\mathrm{kg} / \mathrm{ha})\end{array}$ & $\begin{array}{c}\text { Weed } \\
\text { control } \\
\text { efficiency } \\
(\%)\end{array}$ & $\begin{array}{c}\text { Weed } \\
\text { index } \\
(\%)\end{array}$ \\
\hline 883.85 & 43.51 & 17.47 \\
\hline 976.72 & 37.58 & 20.65 \\
\hline 1034.85 & 33.86 & 22.01 \\
\hline 857.52 & 45.20 & 18.84 \\
\hline 286.85 & 81.66 & 1.03 \\
\hline 360.52 & 76.96 & 11.11 \\
\hline 578.99 & 63.00 & 12.22 \\
\hline 298.85 & 80.90 & 1.44 \\
\hline 696.52 & 55.48 & 15.02 \\
\hline 1240.19 & 20.74 & 21.60 \\
\hline 248.85 & 84.09 & 0.00 \\
\hline 1564.85 & 0.00 & 58.80 \\
\hline 46.65 & - & - \\
\hline 136.83 & - & - \\
\hline 10.74 & - & - \\
\hline
\end{tabular}

( $\mathrm{T}_{2} \mathrm{fb}$ IC and HW at $\left.30 \mathrm{DAS}\right)$ and $\mathrm{T}_{7}\left(\mathrm{~T}_{3} \mathrm{fb} \mathrm{IC}\right.$ and $\mathrm{HW}$ at 30 DAS). All weed control methods established their superiority over weedy check in respect of seed yield, stover yield and yield attributing characters by virtue of reduced weed competition. Angiras et al., (1991) also reported that herbicidal treatments produce significantly higher seed yield over unweeded check in linseed.

\section{Effect of weed management practices on quality of linseed}

The highest oil yield (310 kg/ha) was recorded with the treatment $\mathrm{T}_{11}$ weed free (IC fb HW at 20 and 40 DAS). The higher oil yield received under this treatment was due to the higher seed yield recorded under this treatment which directly responsible for higher oil yields. There was no significant effect on oil content due to various weed 
management treatments (Table 4). The maximum oil content was observed in $T_{11}$ weed free (IC fb HW at 20 and 40 DAS). This might be due to oil content is mainly a genetic character which cannot be manipulated by agronomic practices. The higher oil yield recorded with two hand weeding may be due to deleterious effect of Weedicides on crop development as well as nutrient supply from soil to plant reported by Husain et al., (2015).

\section{References}

Angiras, N. N., Badiyala, D. and Singh, C. M. (1991). Comparative efficacy of herbicides for weed control in flax (Linum usitatissimum L.), Indian J. Weed Science., 23(3-4): 19-23.

Anonymous (2013). www.wikipedia.org/ linseed.

Friesen, G. H. and Freer, J. S, (1991). The control of broad leaved weeds in linseed. Aspects Appl. Biol., 28: 155160.
Husain, K., Dubey, S. D., Verma, R. C., Tripathi, A. K. and Pandey, R. K. (2015). Effect of weed management with post emergence herbicides on seed yield, net return and oil quality of linseed (Linum usitatissimum L.). Current Advances in Agricultural Sciences, 7(2): 120-124.

Linnaeus, C. (1857). Species Plantarum. The Royal Society of London, London, UK, pp: 300.

Mani, V. S., K. C. Gautam and T. K. Chakraborty, 1968. Losses in crop yield due to weed growth. PANS (C). 14: pp. 23-27.

Millam, S., Bohus, O. and Anna, P. (2005).Plant cell and biotechnology studies in Linum usitatissimum- A review. Plant Cell Tissue Organ Cult, 82: 93-103.

Tomar, S. S., Baijpal, R. P. and Bisen, S. V. (1990). Chemical weed control in linseed. Indian journal of Weed Science,22: pp.23-27.

\section{How to cite this article:}

Chhaganiya, H.J., A.P. Pate and Patel, V. D. 2018. Efficacy of Cultural Method of Weed Control on Growth, Yield and Quality of Linseed (Linum usitatissimum L.). Int.J.Curr.Microbiol.App.Sci. 7(02): 2711-2717. doi: https://doi.org/10.20546/ijcmas.2018.702.329 